\title{
Erratum to: Low dose of hydroxychloroquine reduces fatality of critically ill patients with COVID-19
}

\author{
Bo $\mathrm{Yu}^{1}$, Chenze $\mathrm{Li}^{1}$, Peng Chen ${ }^{1}$, Ning Zhou ${ }^{1}$, Luyun Wang ${ }^{1}, \mathrm{Jia} \mathrm{Li}^{2}$, Hualiang Jiang ${ }^{2,3}$ \& \\ Dao-Wen Wang ${ }^{1 *}$ \\ ${ }^{1}$ Division of Cardiology, Department of Internal Medicine and Hubei Key Laboratory of Genetics and Molecular Mechanisms of Cardiological \\ Disorders, Tongji Hospital, Tongji Medical College, Huazhong University of Science and Technology, Wuhan 430030, China; \\ ${ }^{2}$ State Key Laboratory of Drug Research, Shanghai Institute of Materia Medica, Chinese Academy of Sciences, Shanghai 201203, China; \\ ${ }^{3}$ Shanghai Institute for Advanced Immunochemical Studies and School of Life Science and Technology, Shanghai Tech University, Shanghai \\ 201210, China
}

Received May 29, 2020; accepted June 4, 2020; published online June 18, 2020

Erratum to: Sci China Life Sci, 2020, https://oi.org/10.1007/s11427-020-1732-2

Citation: Yu, B., Li, C., Chen, P., Zhou, N., Wang, L., Li, J., Jiang, H., and Wang, D.W. (2020). Erratum to: Low dose of hydroxychloroquine reduces fatality of critically ill patients with COVID-19. Sci China Life Sci 63, 1617-1618. https://doi.org/10.1007/s11427-020-1751-3

1. In the abstract, we missed a piece of information. The correct sentence should be "In this retrospective study, we included 550 critically ill COVID-19 patients who need mechanical ventilation (63.5\%) and oxygen therapy (35.6\%) in Tongji Hospital, Wuhan, from February 1, 2020 to April 4, 2020.”

2. We mistakenly put an approval number from Tongji Hospital ethics committee in the paper (IRBID: TJ-C20200113). The correct number should be TJ-IRB20200229.

3. We mistakenly filled some data in Table 1 and the correct Table 1 (the corrected data are in boldface) should be as follows:

Table 1 Baseline characteristics of critically ill COVID-19 patients ${ }^{\mathrm{a}}$

\begin{tabular}{|c|c|c|c|c|}
\hline & $\begin{array}{c}\text { All patients } \\
(n=550)\end{array}$ & $\begin{array}{c}\text { HCQ } \\
(n=48)\end{array}$ & $\begin{array}{c}\text { NHCQ } \\
(n=502) \\
\end{array}$ & $P$ \\
\hline Age, years & $68(59-77)$ & $68(60-75)$ & $68(59-77)$ & 0.619 \\
\hline \multicolumn{5}{|l|}{ Age range, years } \\
\hline$<\mathbf{6 0}(\%)$ & $139(25.3)$ & $11(22.9)$ & $128(25.5)$ & 0.694 \\
\hline$\geq 60(\%)$ & $411(74.7)$ & $37(77.1)$ & $374(\mathbf{7 4 . 5})$ & 0.694 \\
\hline Gender, male (\%) & $344(62.5)$ & $32(66.7)$ & $312(62.2)$ & 0.537 \\
\hline \multicolumn{5}{|l|}{ Original comorbidities } \\
\hline Hypertension (\%) & $252(45.8)$ & $23(47.9)$ & $229(45.6)$ & 0.760 \\
\hline Coronary heart disease $(\%)$ & $59(10.7)$ & $2(4.2)$ & $57(11.4)$ & 0.147 \\
\hline COPD $(\%)$ & $16(2.9)$ & $0(0)$ & $16(3.2)$ & 0.383 \\
\hline Diabetes $(\%)$ & $94(17.1)$ & $12(25.0)$ & $82(16.3)$ & 0.128 \\
\hline
\end{tabular}

(To be continued on the next page)

*Corresponding author (email: dwwang@tjh.tjmu.edu.cn) 


\begin{tabular}{|c|c|c|c|c|}
\hline & $\begin{array}{l}\text { All patients } \\
(n=550)\end{array}$ & $\begin{array}{c}\text { HCQ } \\
(n=48)\end{array}$ & $\begin{array}{c}\text { NHCQ } \\
(n=502)\end{array}$ & $P$ \\
\hline \multicolumn{5}{|l|}{ Vital signs } \\
\hline Body temperature, ${ }^{\circ} \mathrm{C}$ & $36.7(36.3-37.3)$ & $36.7(36.2-37.3)$ & $36.7(36.3-37.3)$ & 0.704 \\
\hline Pulse, beats $\min ^{-1}$ & $92(80-106)$ & $91(80-103)$ & $92(81-107)$ & 0.594 \\
\hline Respiratory rate, breaths $\min ^{-1}$ & $21(20-26)$ & $25(20-30)$ & $21(20-26)$ & 0.052 \\
\hline Systolic blood pressure, $\mathrm{mmHg}$ & $133(118-148)$ & $131(117-149)$ & $133(118-148)$ & 0.789 \\
\hline Diastolic blood pressure, $\mathrm{mmHg}$ & $80(71-88)$ & $79(70-90)$ & $80(71-88)$ & 0.608 \\
\hline $\mathrm{SpO}_{2}$ on admission $(\%)$ & $95(88-98)$ & $95(90-96)$ & $96(88-98)$ & 0.216 \\
\hline \multicolumn{5}{|l|}{ Symptoms, number/total number (\%) } \\
\hline Fever & $354 / 458(77.3)$ & $29 / 43(67.4)$ & $325 / 415(78.3)$ & 0.105 \\
\hline Cough & $312 / 458(68.1)$ & $29 / 43(67.4)$ & $283 / 415(68.2)$ & 0.920 \\
\hline Sputum production & $223 / 458(48.7)$ & $26 / 43(60.5)$ & $197 / 415(47.5)$ & 0.105 \\
\hline Chest tightness & $65 / 458(14.2)$ & $7 / 43(16.3)$ & $58 / 415(14.0)$ & 0.680 \\
\hline Shortness of breath & $221 / 458(48.3)$ & $30 / 43(69.8)$ & $191 / 415(46.0)$ & 0.003 \\
\hline Nasal congestion & 4/458 (0.9) & $0 / 43(0)$ & $4 / 415(1.0)$ & 1 \\
\hline Nausea & $18 / 458(3.9)$ & $1 / 43(2.3)$ & $17 / 415(4.1)$ & 1 \\
\hline Diarrhea & $100 / 458(21.8)$ & 9/43 (20.9) & $91 / 415(21.9)$ & 0.880 \\
\hline Muscle aches & $32 / 458(\mathbf{7 . 0})$ & $1 / 43(2.3)$ & $31 / 415(7.5)$ & 0.344 \\
\hline Pharynx discomfort & $16 / 458(3.5)$ & $2 / 43(4.7)$ & $14 / 415(3.4)$ & 0.655 \\
\hline Fatigue & $87 / 458(19.0)$ & $8 / 43(18.6)$ & $79 / 415(19.0)$ & 0.945 \\
\hline \multicolumn{5}{|l|}{ Laboratory parameters } \\
\hline White-cell count, $\times 10^{9} \mathrm{~L}^{-1}$ & $7.7(5.5-11.4)$ & $7.3(5.3-12.1)$ & $7.7(5.5-11.4)$ & 0.923 \\
\hline Lymphocyte count, $\times 10^{9} \mathrm{~L}^{-1}$ & $0.7(0.5-1.0)$ & $0.7(0.5-1.0)$ & $0.7(0.5-1.0)$ & 0.923 \\
\hline Neutrophil count, $\times 10^{9} \mathrm{~L}^{-1}$ & $6.2(4.2-10.1)$ & $6.3(4.2-9.5)$ & $6.2(4.2-10.2)$ & 0.909 \\
\hline Platelet count, $\times 10^{9} \mathrm{~L}^{-1}$ & $182.0(129.8-255.0)$ & $189.0(137.0-257.0)$ & $182.0(128.5-255.0)$ & 0.879 \\
\hline Hemoglobin, $\mathrm{g} \mathrm{L}^{-1}$ & $125.0(109.0-139.0)$ & $121.0(107.5-134.5)$ & $125.0(110.0-139.0)$ & 0.424 \\
\hline Alanine aminotransferase, $\mathrm{U} \mathrm{L}^{-1}$ & $26.0(16.0-41.0)$ & $30.0(22.0-52.0)$ & $25.0(16.0-40.0)$ & 0.009 \\
\hline Aspartate aminotransferase, $\mathrm{U} \mathrm{L}^{-1}$ & $35.0(23.0-53.0)$ & $42.0(20.0-60.0)$ & $34.0(24.0-52.0)$ & 0.468 \\
\hline Total bilirubin, $\mu \mathrm{mol} \mathrm{L}^{-1}$ & $10.8(7.8-15.7)$ & $11.0(8.2-15.5)$ & $10.7(7.7-15.8)$ & 0.938 \\
\hline Albumin, $\mathrm{g} \mathrm{L}^{-1}$ & $31.4(28.7-34.5)$ & $31.1(29.6-33.6)$ & $31.5(28.7-34.6)$ & 0.527 \\
\hline Lactate dehydrogenase, $\mathrm{U} \mathrm{L}^{-1}$ & $395.5(293.0-536.0)$ & $401.0(311.5-493.0)$ & $395.0(291.5-541.0)$ & 0.949 \\
\hline Creatinine, $\mu \mathrm{mol} \mathrm{L}^{-1}$ & $81.0(62.0-103.0)$ & $86.0(63.0-110.5)$ & $79.0(62.0-103.0)$ & 0.440 \\
\hline Blood urea nitrogen, $\mathrm{mmol} \mathrm{L}^{-1}$ & $6.4(4.4-10.4)$ & $5.9(4.1-9.6)$ & $6.5(4.5-10.7)$ & 0.392 \\
\hline International normalized ratio & $1.1(1.1-1.3)$ & $1.1(1.1-1.2)$ & $1.2(1.1-1.3)$ & 0.034 \\
\hline D-dimer, $\mathrm{mg} \mathrm{L}^{-1}$ & $2.3(1.1-9.6)$ & $2.6(1.0-10.1)$ & $2.3(1.1-9.7)$ & 0.895 \\
\hline APTT, s & $40.3(36.6-45.4)$ & $39.0(36.2-50.7)$ & $40.4(36.6-44.9)$ & 0.962 \\
\hline C-reactive protein, $\mathrm{mg} \mathrm{L}^{-1}$ & $75.7(36.3-128.9)$ & $84.1(27.5-117.4)$ & $75.6(37.1-131.5)$ & 0.789 \\
\hline NT-ProBNP, pg mL ${ }^{-1}$ & $598.5(223.5-1814.3)$ & $600.5(191.8-1926.5)$ & $598.5(224.5-1829.8)$ & 0.803 \\
\hline cTnI, pg mL $\mathrm{m}^{-1}$ & $15.9(6.2-59.5)$ & $12.1(6.5-47.2)$ & $16.3(6.2-60.7)$ & 0.582 \\
\hline IL-6, pg mL ${ }^{-1}$ & $31.4(14.0-96.1)$ & $25.3(12.0-111.1)$ & $31.6(14.0-95.9)$ & 0.621 \\
\hline IL-10, pg mL ${ }^{-1}$ & $6.5(5.0-12.7)$ & $6.8(5.0-9.3)$ & $6.5(5.0-13.5)$ & 0.706 \\
\hline IL-8, pg mL ${ }^{-1}$ & $22.4(12.0-43.8)$ & $23.1(10.4-30.8)$ & $22.2(12.1-47.5)$ & 0.471 \\
\hline TNF- $\alpha, p g \mathrm{~mL}^{-1}$ & $10.6(8.2-14.6)$ & $11.0(9.1-13.7)$ & $10.4(8.2-14.8)$ & 0.596 \\
\hline IL-1 $\beta$, pg $\mathrm{mL}^{-1}$ & $5.0(5.0-5.0)$ & $5.0(5.0-5.3)$ & $5.0(5.0-5.0)$ & 0.524 \\
\hline $\mathrm{IL}-2 \mathrm{R}, \mathrm{U} \mathrm{mL} \mathrm{m}^{-1}$ & $1026.0(679.0-1501.0)$ & $977.5(749.0-1544.8)$ & $1026.0(672.0-1490.5)$ & 0.592 \\
\hline Oxygen therapy, number (\%) & $545(99.1)$ & 47 (97.9) & $498(99.2)$ & 0.368 \\
\hline Mechanical ventilation, number (\%) & $349(63.5)$ & $28(58.3)$ & $321(63.9)$ & 0.441 \\
\hline
\end{tabular}

a) Mechanical ventilation contained non-invasive ventilation and invasive ventilation. Data are presented as medians and interquartile range (Q1-Q3). HCQ, hydroxychloroquine treatment; NHCQ, non-hydroxychloroquine treatment; COPD, chronic obstructive pulmonary disease; APTT, activated partial thromboplastin time; NT-proBNP, N-terminal pro-B-type natriuretic peptide; cTnI, cardiac troponin I; IL, interleukin; TNF- $\alpha$, tumor necrosis factor- $\alpha$; $\mathrm{SpO}_{2}$, percutaneous oxygen saturation.

The online version of the original article can be found at https://doi.org/10.1007/s11427-020-1732-2 